\title{
Optimal Allocation of Vehicles to Bus Routes Using Automatically Collected Data and Simulation Modelling
}

Keywords (up to 8):

fleet allocation, vehicle allocation, running time variability, simulation, bus transit, public transportation

Classification codes (up to 6):

L920

\section{ABSTRACT}

Monitoring the service quality of high-frequency bus transit is important both to agencies running their own operations and those contracting out, where performance measures can be used to assess contract penalties or bonuses. The availability of automatically collected vehicle movement and demand data enables detecting changes in running times and demand, which may present opportunities to improve service quality and fleet utilization. This research develops a framework to maximize service performance in a set of highfrequency bus routes, given their planned headways and a total fleet size constraint. Using automatically collected data and simulation modelling to evaluate the performance of each route with varying fleet sizes, a greedy algorithm adjusts allocation toward optimality. A simplified case study involving morning peak service on nine bus routes in Boston demonstrates the feasibility and potential benefits of the approach. A potential application is automated detection of routes operating with insufficient or excessive resources.

\section{Introduction}

Most likely, the management of a transit agency has, through careful service planning and performance monitoring, set the fleet size on most routes at an appropriate level. However, the nature of this ongoing task places more emphasis on problem routes; for instance, routes that are suffering delays due to roadwork or routes that are heavily crowded. Management will receive more complaints from passengers using these services, or it might have the initiative to revise resource levels in anticipation of such problems. In the meantime, there may be undetected opportunities to save resources on routes that are performing well and have excess resources, as well as to improve service performance of routes that could greatly benefit from added resources but have not yet received special attention. This research addresses the problem of assigning vehicles to routes of a bus network, given a target headway for each route and a fixed total fleet size, with the objective of maximizing total service performance.

There are a number of factors that affect service performance. Some are in control of the operator at the time of operation, such as the dispatching discipline used at terminals and the set of strategies used to regulate headways. Others, like infrastructure, congestion, and demand for service are considered fixed in the short term. Still others, like scheduled cycle time, are in control of management at the service planning stage. Greater cycle times result in greater recovery times at terminals, thereby making it easier to dispatch vehicles regularly. In the service planning stage, increasing cycle times is an effective strategy to manage running time variability. 
Cycle time $c$ is determined by target headway $h$ and fleet size $n$, through the relationship $c=n h$. Greater cycle times can be achieved by increasing fleet size, increasing headway, or a combination of both. Increasing headway while holding fleet size constant increases cycle time without additional operating cost, but it decreases the passenger carrying capacity of the service, making vehicles more crowded, in addition to increasing passenger waiting times. Although the scheduled cycle time will have increased, dwell times at stops will increase and become more variable, so the net effect could very well be lower service quality if ridership is high. When ridership is relatively low and the operator is struggling to dispatch vehicles at regular intervals, the additional recovery time provided by an increased headway can improve service reliability.

An alternative way to increase cycle times is to increase fleet size while holding the target headway constant. This does increase operational cost, but it adds slack to running times without directly affecting waiting times or loads. If the operator uses the additional resources correctly, headway regularity can improve, which in turn decreases waiting times and balances loads from vehicle to vehicle. Balanced loads can, in turn, reduce running time variability through less variable dwell times. The extra slack can be used at the terminals to improve dispatch regularity, but also en route in the form of holding or slowing vehicles strategically to maintain regular headways. In the latter case, running time variability decreases at the expense of higher typical running times. Increasing fleet size is perhaps the most effective way of dealing with running time variability at the service planning stage.

Of all the factors that affect service quality, only fleet size will be considered in this research. Headway determination responds to a wide range of factors, including policy and network effects. Simultaneous consideration of headway and fleet size would certainly be a more complete approach to optimizing resource allocation, but also a more complex one. The premise of this research is that the target headway of each route has been appropriately set based on service delivery policy and demand for service. The objective is to allocate resources in a way that maximizes service quality in the current set of routes, without altering the service plan.

In this research, optimization is used to allocate a fixed total amount of resources among a group of routes, without changing their frequencies or operating strategies, in a way that maximizes service performance. Optimization has played an important role in planning, frequency determination, and vehicle and crew scheduling for bus transit (Desaulniers, 2007; Ibarra-Rojas et. al., 2015). The optimization approach followed in this research differs from many past approaches in that it optimizes the allocation of a fixed total amount of vehicles over a group of routes rather than determining frequencies, although the two problems are closely related (Ceder, 1984; Constantin and Florian, 1995; Furth and Wilson, 1981; Li et. al., 2013; Martínez et. al., 2014; Verbas and Mahmassani, 2013; Yu et. al., 2010). Han and Wilson (1982) focus on allocation of vehicles in the case of heavily utilized overlapping routes.

This paper is organized as follows: Section 2 presents a framework to optimize resource allocation and Section 3 discusses a specific optimization method. The problem is solved separately for each time period (e.g. first the morning peak, then the mid-day, then the afternoon peak, etc.) One of the challenges faced is the systematic variation of running 
times and headways within each of these time bands. An operator will vary the fleet size of a route according to this, providing the peak vehicle requirement of each time period only when it is needed. Since schedule data is not available for the hypothetical scenarios with smaller or larger fleets, vehicle profiles must be estimated. Section 4 discusses this further and proposes heuristics to overcome the obstacle. Section 5 presents a simple application of the framework and optimization algorithm to a set of twelve bus routes. Section 6 closes with a summary and concluding remarks.

\section{Framework}

Increasing the fleet size of a bus service can enhance an operator's ability to maintain regular headways, thereby improving service performance. It is in everyone's interest to have good performance, but adding resources is feasible only up to the overall fleet size (or budget) constraint. Optimizing resource allocation involves identifying where resources are needed the most. The objective is to find the resource allocation that attains the best overall service performance under an existing service plan, present operating conditions, and specified overall fleet size.

\subsection{Service Performance}

Service performance is a general term that can capture many quantifiable aspects of bus operations. The primary factors relate to service quality as perceived by passengers. For example, performance may include measures of waiting time and crowding. These may be expressed in absolute terms or relative to a performance standard. For example, the average number of minutes of wait experienced by passengers is an absolute measure of waiting time, while excess waiting time is expressed relative to the headway specified in the service plan. Performance measures based on vehicles (rather than passengers), such as the standard deviation of headways, can also be included. Moreover, service performance can capture general aspects of operations, such as congestion at terminals due to excess number of vehicles standing.

When multiple criteria are driving the optimization process, the relative importance of each factor must be considered. There may be a situation in which adding a vehicle to one route improves one aspect of service performance considerably while not significantly affecting a second aspect. Adding the vehicle to a different route instead may considerably improve the second aspect of service performance while not affecting the first very much. In such cases, the trade-off between the two aspects must be considered in deciding to which route the vehicle should be allocated.

The performance improvement resulting from adding a vehicle to a route will decrease as fleet size increases. When a route is severely under-resourced, the addition of one vehicle can have a profound impact on service performance. In contrast, when a route has excess resources, the addition of one vehicle might not have visible effects on performance.

Figure 1 illustrates the conceptual relationship between fleet size and the performance of a route. There are four regimes in this relationship, separated by dotted vertical lines. At least one vehicle must be allocated to provide service. Once service is being provided, additional vehicles will bring about performance improvements, albeit at a decreasing rate. The rate 
will continue to decrease until adding a vehicle does not result in visible performance improvements. While there are no capacity problems as a result of a large fleet size, continuing to add vehicles will not affect performance. However, having an excess number of vehicles can degrade performance if, for instance, there is insufficient capacity at terminals for vehicles to stand and vehicle congestion at terminals is one of the performance factors under consideration. The exact shape of the performance function depends on the factors composing it and the way operators manage the different levels of resource. However, it is hypothesized that many practical performance functions will be concave, as shown in Figure 1.

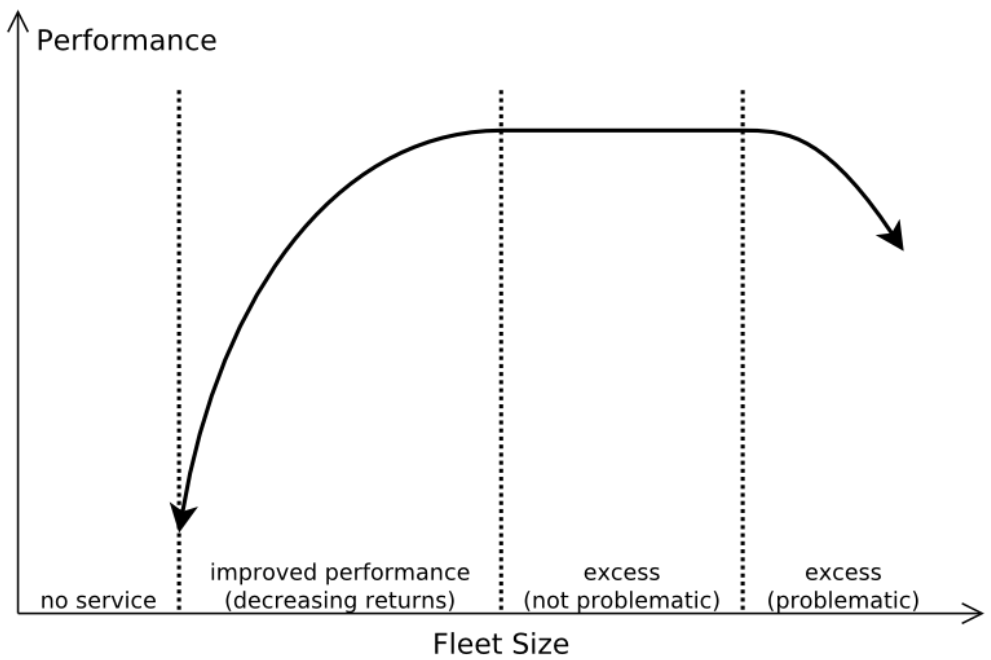

Figure 1. General relationship between performance and fleet size

Factors such as waiting time and loads should decrease as resources increase, as long as the operator behaviour is consistent across allocations. These factors are negative in the sense that, for example, higher waiting times imply lower performance. If a positive performance function is desired, these factors must be transformed somehow (for example, by taking negative waiting time as a measure of performance). If all aspects of performance being considered are negative, an alternative is to include them untransformed in the performance function. In this case, the performance function will be convex rather than concave, with lower values of it signifying better performance.

Were the objective to maximize performance of a single route, the optimal allocation would add vehicles until performance stops improving or until all available vehicles have been allocated, whichever comes first. In the absence of resource constraints, the same logic applied independently to each route would yield optimum performance over a set of routes. However, the resulting allocation may not be feasible when total available resources are constrained. In this case, resources should be first allocated where they yield the largest performance benefit, until the total available resources are exhausted.

\subsection{Existing Service Plan and Operating Conditions}

As stated earlier, the objective is to find the optimum resource-constrained allocation under an existing service plan and operating conditions. Since the matter in question is how performance responds to changes in fleet size, fleet size will be varied while other factors 
remain controlled. Route alignment, span of service, and specified headway by time of day (all of which form part of the service plan) will be held constant. Moreover, operating conditions such as levels of traffic, road geometry, and ridership patterns will be assumed constant. The implication is that running times (possibly excluding dwell times) will remain constant, independent of fleet size.

The effect of changing the fleet size of a route will be partially governed by operating behaviour. For instance, if dispatching at terminals is based on a target headway, the mean headway will not fall below the specified headway, regardless of how many vehicles are allocated. On the other hand, if terminal dispatching is based on even headways, the specified headway does not come directly into play. In this case, observed mean headway may decrease below the specified headway if the resource allocation is high enough. This implies, in effect, a simultaneous change in fleet size and operating headway, which is not the intent of this framework.

A key assumption of this optimization framework is that the current operating conditions will not change as a result of changes in fleet size. This assumption may be valid only under small changes in resource allocation and in the short run. Large changes in fleet size may attract new ridership or drive away current passengers to alternative routes or modes, especially if a common corridor is shared among multiple routes. It would be possible to capture these effects with corridor- or network-level ridership models, but this falls outside the scope of this research. Instead, a limit on the maximum change in fleet size per route can be imposed. For example, adjustments of up to two vehicles added or removed could be considered.

\subsection{Optimization Model Formulation}

The optimization problem takes the following form:

$$
\begin{aligned}
\text { maximize } & \sum_{r \in R} Q_{r}\left(x_{r}\right) \\
\text { s.t. } & \sum_{r \in R} x_{r}=n \\
& x_{r} \in \mathbb{Z}^{+} \forall r \in R
\end{aligned}
$$

where $R$ denotes the set of routes under consideration, $Q_{r}\left(x_{r}\right)$ is the performance function of the $r^{\text {th }}$ route, $x_{r}$ is the number of vehicles assigned to route $r$, and $n$ is the total fleet size. $Q_{r}\left(x_{r}\right)$ captures the service performance of route $r$ when it operates with $x_{r}$ vehicles, using a linear combination of performance measures. The function can represent performance measures such as expected waiting time and in-vehicle crowding, with weights reflecting the relative importance of each. For example, it could take the following form:

$$
Q_{r}=-(5 \cdot \mathrm{EWT}+\mathrm{EL})
$$

where EWT is the route-level excess waiting time in minutes and EL is the route-level excess load, some measure of in-vehicle crowding measured in units of passengers or passengerstops. The 5 preceding EWT means that an excess load of five passengers is as onerous as one minute of excess waiting time. The sign of the terms is reversed to make them positive, 
such that higher values of $Q_{r}$ indicate better performance. The set of routes in $R$ can range from one route to all routes in a network. It can contain only routes belonging to the same operator, or routes based on the same depot, in case it is desirable to maintain the current total resource levels on an operator or depot basis. Constraint (1b) ensures that the sum of individual route fleet sizes equals the total available fleet size, while constraint (1c) limits vehicle allocations to (strictly) positive integers.

More constraints can be added to enrich the model formulation. A limit on the maximum change in fleet size of each route can be imposed. Such a constraint is necessary for the assumption of a constant operating environment independent of fleet size to remain valid, as discussed in Section 2.2. To allow a maximum change of $\pm \Delta_{\max }$ vehicles, the following constraint is added:

$$
\left|x_{r}-x_{r, 0}\right| \leq \Delta_{\max }
$$

where $x_{r, 0}$ denotes the original allocation of vehicles assigned to route $r$ and $\Delta_{\max }$ is the maximum allowable change. Setting $\Delta_{\max }=2$ limits changes to \pm 2 vehicles.

As discussed in Section 2.2, it may be desirable to avoid allocating an excessive amount of vehicles to a route given the running time distribution and specified headway $h_{r}$, since this may allow an operator to serve the route at a frequency higher than specified (which implies simultaneous changes in fleet size and frequency). Scheduled cycle time can be limited to $c_{r \text {, max }}$ by imposing the following constraint:

$$
x_{r} \leq \frac{c_{r, \max }}{h_{r}}
$$

The cycle time limit can be determined based on a maximum allowable percentile of running times. For example, $c_{r \text {, max }}$ could be set to the sum of $95^{\text {th }}$ percentile running times in each direction. Similarly, it may be desirable to impose a minimum acceptable performance level. To enforce a minimum of some aspect of performance $q$, a constraint of the form

$$
q_{r} \geq L_{r} \forall r \in R
$$

is included in the formulation, where $q_{r}$ is a particular component of $Q_{r}$ and $L_{r}$ is its lower limit. This is primarily applicable to positive factors of performance. For negative factors, an upper limit is appropriate:

$$
q_{r} \leq U_{r} \forall r \in R
$$

where $U_{r}$ is an upper limit of $q_{r}$. For example, a constraint of the form $\mathrm{EWT}_{r} \leq 5$ would prevent an excess waiting time above five minutes. This same type of constraint can be used in cases where there is concern that the addition of vehicles to a route given by the optimal solution (in terms of service quality criteria) leads to a congestion problem at the terminal because the physical limit on the amount of vehicles that can be standing simultaneously is exceeded. This is achieved by letting $q_{r}$ in (6) be some measure of congestion. Care should be exercised with constraints (5) and (6), since they can make the problem infeasible. For example, it may not be possible to decrease excess waiting time beyond some limit, regardless of fleet size, due to operating conditions such as exogenous running time variability. 
It is possible to penalize some aspect of service performance without explicitly constraining it. For example, a penalty can be imposed on exceeding the capacity of a terminal for standing vehicles. To do so, the objective function takes the following form:

$$
\text { maximize } \sum_{r \in R}\left(Q_{r}\left(x_{r}\right)-P_{r}\left(x_{r}\right)\right)
$$

where $P_{r}\left(x_{r}\right)$ is a penalty due to exceeding terminal capacity. For instance, $P_{r}\left(x_{r}\right)$ might be the duration in minutes of instances where more vehicles than the specified limit are standing simultaneously, multiplied by a coefficient to weigh the penalty against the different performance criteria. This type of penalty is sometimes called a soft constraint.

\section{Optimization Method}

\subsection{Solution Framework}

Three processes work together towards the optimal solution:

1. an optimizer allocates $x_{r}$ vehicles to route $r \in R$, subject to a set of constraints, and adjusts this allocation in search of better performance;

2. a vehicle profiler determines vehicle profiles (i.e. the number of available vehicles by time of day) given the allocation specified by the optimizer; and

3. a simulator models operations of route $r$ with a given vehicle profile and estimates the performance measures with which the objective function is evaluated.

This research focuses on the optimization process, and assumes that vehicle profiler and simulator algorithms as described above are available. The simulation model employed should be as realistic as possible in terms of running times, dwell times, operator behaviour, and ridership patterns. Simulation gives estimates of performance measures, which come from a stochastic environment. The confidence intervals of these estimates should be made reasonably tight by simulating operations for a sufficiently long period. This research uses a simulation model based on the framework developed by Sánchez-Martínez (2012). Devising a realistic vehicle profiler to translate $x_{r}$ to a vehicle profile is a non-trivial aspect of the problem, which is discussed further in Section 4.

The allocation problem is solved independently for each time period, so it is required that a single set of common time periods is used for all routes. A few reasonably long time periods should be used rather than many short ones; this is because the resulting number of vehicles assigned to each route can vary across time periods, and a solution would not be practical if this fluctuated, for instance, every 30 minutes. The allocation problem should be solved for the first time period first, then for the second, etc., because previous time periods dictate the initial conditions of the time period being analysed.

The objective is to maximize total performance, which in the formulation is the sum of performance functions of all routes. In the absence of restrictions on the shape of performance functions, their sum can take on an arbitrary shape, and the only way of finding the (globally) optimal allocation is to enumerate all feasible solutions. It was argued in Section 2.1, however, that most practical specifications of performance functions should be concave. Assuming this is the case, the objective function is also concave, so it has only one 
local maximum, which is also the global maximum. This has an important implication: if at some point in the optimization process there is no way to improve the solution by taking a local step, that solution is guaranteed to be globally optimal. The concavity assumption can be checked during the optimization process, and if a violation is detected, a more thorough analysis can be made.

The optimization procedure is broken down into two stages. The first stage of the procedure deals with obtaining a solution that uses exactly $n$ vehicles. The second stage moves vehicles from one route to another until there are no further changes that can improve service quality. Both stages employ a greedy algorithm that takes discrete local steps of one vehicle. An activity diagram of the method is shown in Figure 2. The result is globally optimal under the global concavity assumption discussed previously.

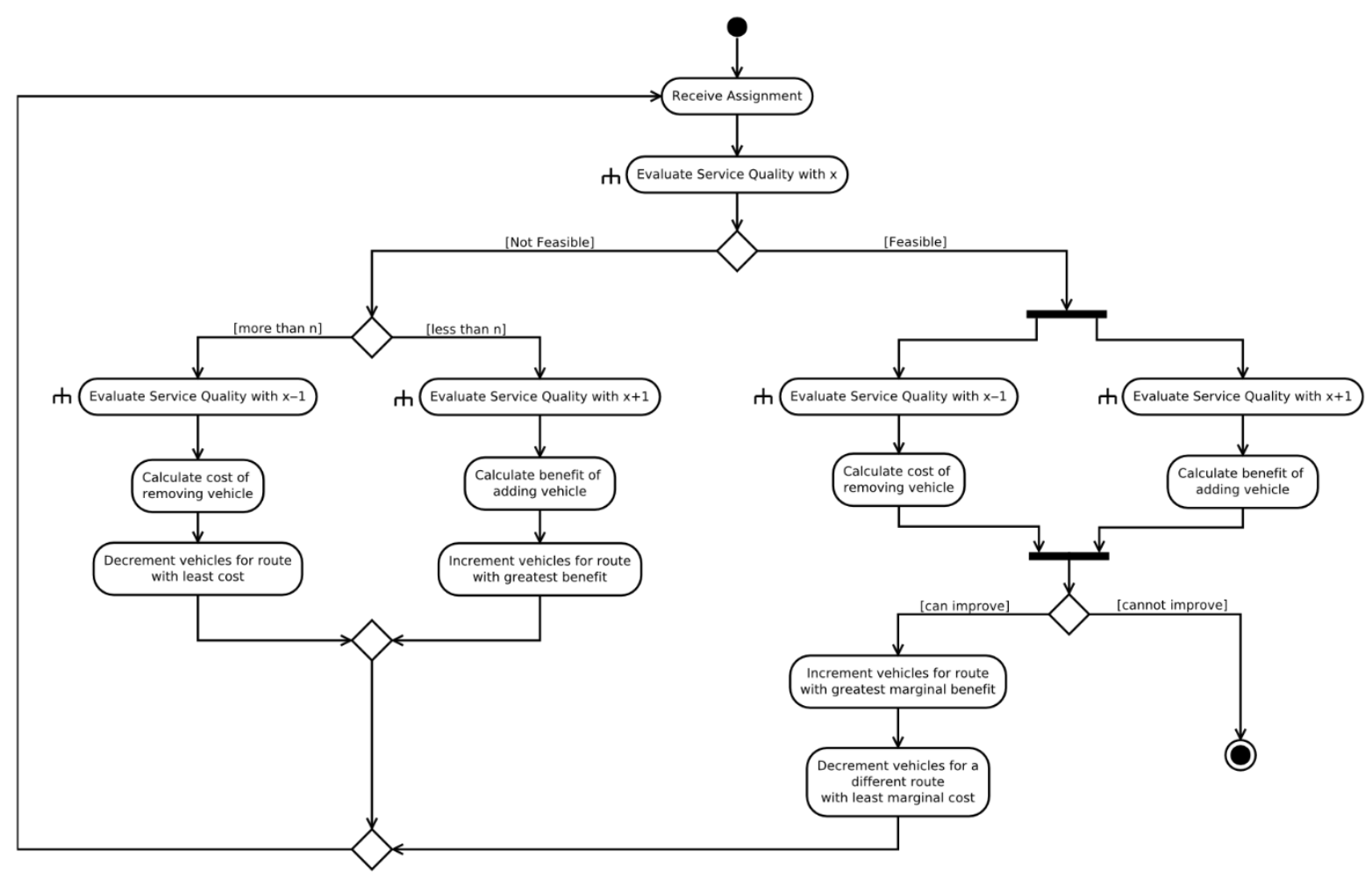

Figure 2: Activity Diagram of Optimization Method

\subsection{Achieving the Target Total Fleet Size}

1. An initial solution is obtained, either by setting each $x_{r}$ to the current values or by choosing positive values arbitrarily. The solution need not meet constraint (1b) but must be otherwise feasible.

2. $Q_{r}\left(x_{r}\right)$ is evaluated with the current assignments $x_{r}$.

3. This step is carried out only if there are fewer than $n$ vehicles assigned.

a. $Q_{r}\left(x_{r}+1\right)$ is evaluated for all routes.

b. The marginal benefit of adding a vehicle to route $r$, denoted by $\delta_{r}^{+}$, is determined for every route.

$$
\delta_{r}^{+} \leftarrow Q_{r}\left(x_{r}+1\right)-Q_{r}\left(x_{r}\right)
$$

Let $r_{*}^{+}$denote the route with maximum $\delta_{r}^{+}$. 


$$
r_{*}^{+}=\operatorname{argmax}_{r \in R}\left\{\delta_{r}^{+}\right\}
$$

c. One vehicle is added to the route with greatest marginal benefit.

$$
x_{r_{*}^{+}} \leftarrow x_{r_{*}^{+}}+1
$$

d. Step 3 is repeated until the number of vehicles assigned equals the target fleet size $n$. At this point, the solution is feasible and the algorithm continues with the steps of Section 3.3.

4. This step is carried out only if there are more than $n$ vehicles assigned.

a. $Q_{r}\left(x_{r}-1\right)$ is evaluated for all routes.

b. The marginal cost of removing a vehicle from route $r$, denoted by $\delta_{r}^{-}$, is determined for every route.

$$
\delta_{r}^{-} \leftarrow Q_{r}\left(x_{r}\right)-Q_{r}\left(x_{r}-1\right)
$$

Let $r_{*}^{-}$denote the route with minimum $\delta_{r}^{-}$.

$$
r_{*}^{-}=\operatorname{argmin}_{r \in R}\left\{\delta_{r}^{-}\right\}
$$

c. One vehicle is removed from the route with the least marginal cost.

$$
x_{r_{*}^{-}} \leftarrow x_{r_{*}^{-}}-1
$$

d. Step 4 is repeated until the number of vehicles assigned equals the target fleet size $n$. At this point, the solution is feasible and the algorithm continues with the steps of Section 3.3.

\subsection{Obtaining the Optimal Solution}

Once a feasible solution is obtained, the following steps are followed to arrive at an optimal solution:

1. Both $Q_{r}\left(x_{r}-1\right)$ and $Q_{r}\left(x_{r}+1\right)$ are evaluated for all routes.

2. The marginal benefit of adding a vehicle is determined for every route.

$$
\delta_{r}^{+} \leftarrow Q_{r}\left(x_{r}+1\right)-Q_{r}\left(x_{r}\right)
$$

Likewise, the marginal cost of removing a vehicle is determined for every route.

$$
\delta_{r}^{-} \leftarrow Q_{r}\left(x_{r}\right)-Q_{r}\left(x_{r}-1\right)
$$

Let $r_{*}^{+}$denote the route with greatest marginal benefit of adding a vehicle and $r_{*}^{-}$ denote a different route with least marginal cost of removing a vehicle.

$$
\begin{gathered}
r_{*}^{+}=\operatorname{argmax}_{r \in R}\left\{\delta_{r}^{+}\right\} \\
r_{*}^{-}=\operatorname{argmin}_{r \in R \backslash r_{*}^{+}}\left\{\delta_{r}^{-}\right\}
\end{gathered}
$$

3. If $\delta_{r_{*}^{+}}^{+}>\delta_{r_{*}^{-}}^{-}$, a vehicle is removed from $r_{*}^{-}$and added to $r_{*}^{+}$. This keeps the total number of vehicles at $n$ and increases the objective function value.

$$
\begin{aligned}
& x_{r_{*}^{-}} \leftarrow x_{r_{*}^{-}}-1 \\
& x_{r_{*}^{+}} \leftarrow x_{r_{*}^{+}}+1
\end{aligned}
$$

4. Steps 1-3 are repeated until $\delta_{r_{*}^{+}}^{+} \leq \delta_{r_{*}^{-}}^{-}$. At this point, the solution is optimal and the algorithm terminates. 


\subsection{Maintaining Feasibility}

Feasibility must be maintained throughout the optimization process. If the formulation includes constraints imposing a maximum allowable change in fleet size, a maximum implied cycle time, or lower or upper bounds on some aspect of service performance, only fleet sizes meeting these constraints can be considered. For example, adding a vehicle to route $r$ will not be considered if this causes unacceptable congestion at terminals, even if this allocation increases total performance the most. The next best route becomes the candidate to receive the additional vehicle.

\section{Building a Vehicle Profile}

While the optimization problem deals with a single $x_{r}$ per period, real bus operations may exhibit within-period variation in fleet size. Simulation models are capable of capturing this, but they require a vehicle profile as an input. It is unlikely that an operator will plan to operate with the peak vehicle requirement at times during the peak period that require a lower number of vehicles. Hypothetical schedules for situations with a smaller or larger fleet size are generally not available.

Faced with this reality, we must devise a way of building a vehicle profile given $x_{r}$. The approach used here modifies an existing vehicle profile. There are multiple ways to do this, each with its implications on estimates of service performance depending on the operating strategies modelled. Three heuristics are described below and illustrated in Figure 3. In the illustration, $x_{r}$ is one vehicle less than the number of vehicles currently used. The current vehicle profile is shown with a thin solid line and the vehicle profile assumed under the new allocation $x_{r}$ is indicated by a thick solid line.

1. The simplest strategy is to make all $x_{r}$ vehicles available throughout the entire period. If $x_{r}$ is the peak vehicle requirement for the period, it is likely that the model will operate with more vehicles than in reality (outside this within-period peak), and performance will be overestimated. With even headway dispatching, this means lower waiting times. With target headway dispatching, this leads to a higher probability of adherence to the scheduled headway at terminals, which leads initially to lower waiting times and eventually to excess vehicles queuing at terminals to begin trips.

2. A strategy that tries to incorporate variation of active vehicles within a period is to shift the current vehicle profile by a constant amount throughout the period, namely the adjustment in the peak vehicle requirement. For example, if the peak vehicle requirement being tested is one less than the base case, the entire vehicle profile is shifted down by one vehicle. (The profile must remain strictly positive at all times.) The result may be different from the operator's plan in response to the change in peak vehicle requirement, but it is not clear if performance is over- or underestimated in this case.

3. An alternative strategy that also tries to capture within-period variability in the active route fleet size is to calculate end-to-end running time reliability obtained with $x_{r}$ in the portion of the period with peak vehicle requirement in the base profile, and then calculate the fleet size required to deliver the same running time reliability during the 
rest of the period. For example, an allocation of 20 peak vehicles might be made for the morning peak period of a route, but perhaps all 20 vehicles are only used during the busiest half hour of the period. Given the specified headway $h$ and the fleet size $n$, the cycle time is $c=n h$, which implies, for instance, $90^{\text {th }}$ percentile running times in the peak of the peak. Then the fleet size during the remainder of the morning period is set so that it achieves the same level of reliability. In other words, it is calculated with $n \approx c / h$, with the cycle time determined the $90^{\text {th }}$ percentile of the running time distributions at other times in the period. This strategy may lead to changes in the shape of the base vehicle profile. If the operator bases new operational plans on running time distributions, this strategy may lead to a realistic vehicle profile. This is not necessarily the case, however, since crew and vehicle constraints are not considered.

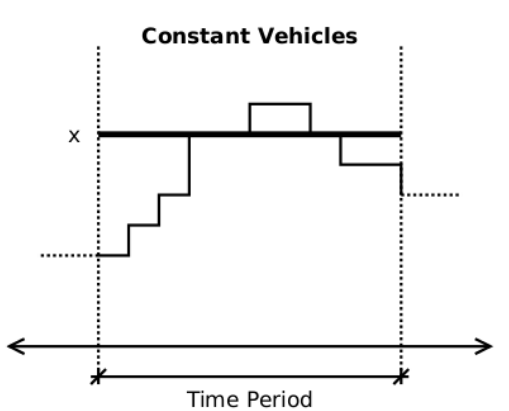

(a)

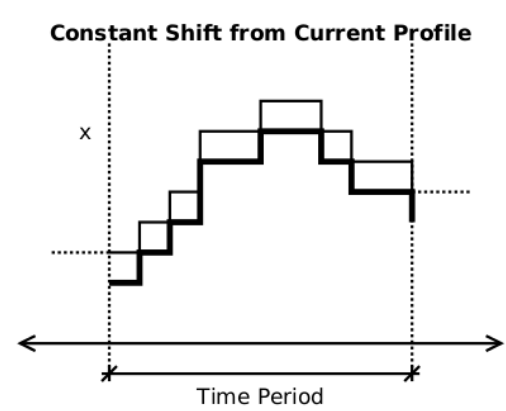

(b)

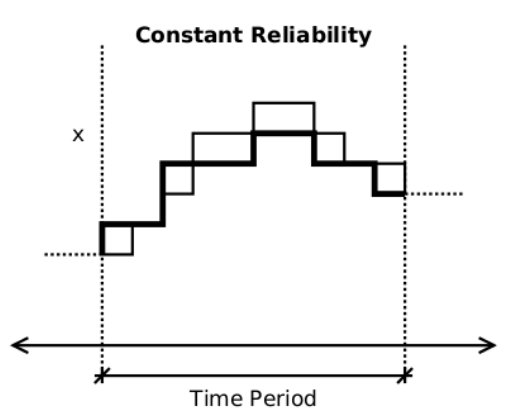

(c)

Figure 3: Three methods for obtaining a vehicle profile from $x_{r}$

An alternative approach is to build an operating plan that meets level-of-service specifications and minimizes operating cost. An optimization package could be used to build vehicle and crew schedules once the time table has been generated. This approach does not depend on an existing vehicle profile, but requires modelling vehicle and crew costs and constraints, a task that falls outside the scope of this research. Moreover, this approach would generate a vehicle profile for the entire day, not just the time period of interest, so the optimization process would not be as separable by time periods as proposed here.

\section{Application}

This section applies the fleet allocation optimization framework to nine high-frequency bus routes of the Massachusetts Bay Transportation Authority (MBTA) in the Boston metropolitan area. The routes selected have mean scheduled headways of less than 10 minutes between 7:30 and 9:00, i.e. during morning peak service on weekdays, and a single run variation (e.g. short and long versions or special trips) per direction.

For simplicity, morning peak service for each route was modelled as a stationary process, i.e. without systematic variation of running times or changes in the number of vehicles operating during the analysis period starting at 7:30 and ending at 9:00. The mean terminal departure scheduled headway was taken as the operating headway for the entire analysis period. The current number of vehicles operating was estimated by $n=\lceil c / h\rceil$. The cycle time assumed for this calculation was obtained by adding the maximum scheduled running times for both 
directions of each route. Maximum, rather than mean, scheduled running times were used in addition to rounding up route fleet sizes because scheduled running times exclude recovery time, although the variation in scheduled running times, if any, was generally small. This resulted in a combined fleet size of 91 vehicles for the set of routes.

Running times were obtained from automated vehicle location (AVL) records of timing point crossings for weekday service between 7:30 and 9:00 during March 2014. Terminals and key stops are designated as timing points by the MBTA. There are an average of 7 timing points per route-direction pair, with shorter routes having fewer timing points and longer routes having more timing points. A probability distribution of running times between timing points was obtained from these observations. These running times include dwell times at stops.

Demand data was obtained from automated fare collection (AFC) records for the same period as AVL. Passengers were not explicitly modelled in simulation. The algorithm by Gordon et.al. (2013) was used to infer origins, destinations, and transfers by analysing the sequence of fare transactions for each card and combining AFC and AVL data. This resulted in a large sample of fare transactions with inferred origin and destination. Demand was scaled up to the observed number of boarding passengers at each stop. Mean passenger arrival rates by origin-destination pair were calculated from this scaled demand. The passenger arrival process was modelled as a Poisson process with the calculated mean arrival rates, a reasonable choice for high-frequency transit services in which passengers do not time their arrivals at stops following a schedule. Passengers were simulated as agents appearing at their origin stop, waiting for a vehicle, boarding the first vehicle with remaining capacity, and alighting at their destination stop. From this process waiting times and loads were observed.

The optimization objective was set to minimize the linear combination of excess waiting time and excess load. Excess waiting time was calculated as the difference between the mean observed waiting time and half the operating headway, measured in minutes. Excess load was calculated as loads in excess of 40 passengers, measured in units of passenger-stops. The two components were weighted equally. Evaluations of this user cost function were based on 10000 hours of simulated operations, excluding a 3 hour warm-up period. The simulation model used a target headway dispatching policy at terminals, with the target headway equal to the mean scheduled headway, and no holding control at stops. The algorithm discussed in Section 3 was employed for optimization, setting the fleet size constraint to keep the total fleet size constant. No other constraints were specified.

Figure 4 shows user cost as a function of fleet size for each route at different stages in the optimization process. Outlined circles mark the initial fleet size, and filled circles mark the final fleet size, for each route. Lines mark each simulated fleet size scenario. Plot (a) shows the initial solution with the current vehicle allocation, and a cost of 2.356. Plot (b) shows the initial solution after decreases and increases of one vehicle have been evaluated through simulation. Steep line segments indicate a relatively large performance benefit from the addition of a vehicle, while near-horizontal line segments indicate a relatively small performance penalty from the removal of a vehicle. Plot (c) illustrates the first iteration of the algorithm, which removes a vehicle from one route and adds a vehicle to another route, while decreasing user cost by $20 \%$. Plot (d) illustrates the second and final iteration, which again 
re-allocates a vehicle from one route to another while decreasing user cost by $17 \%$. After this iteration, no further re-allocation reduces user cost.

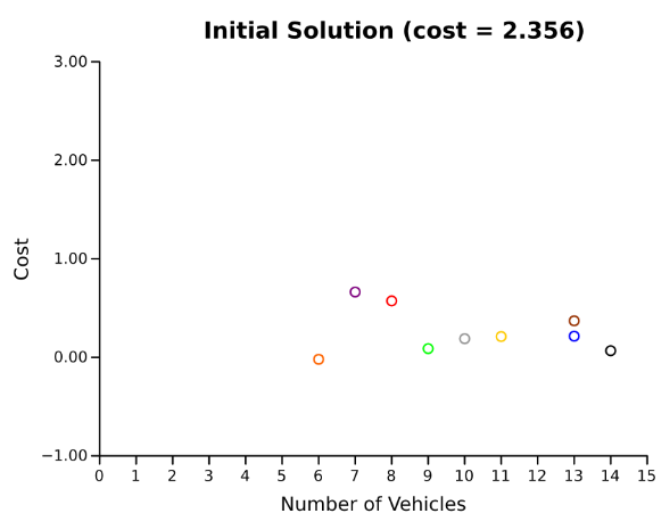

(a)

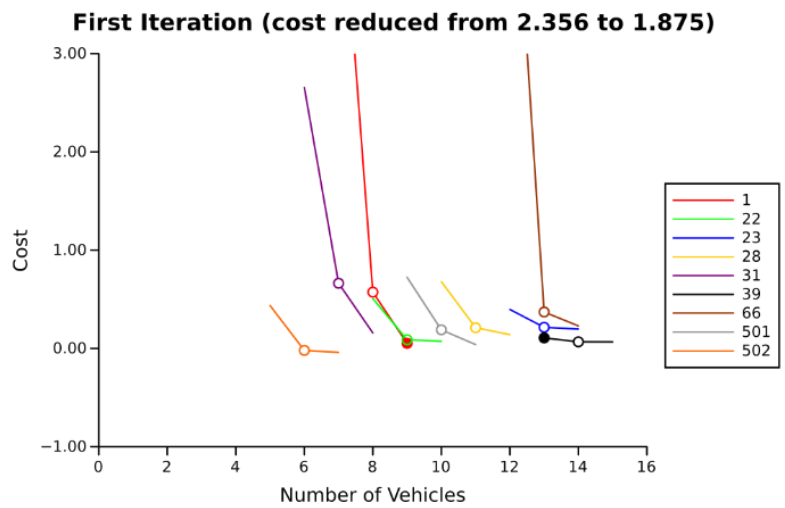

(c)

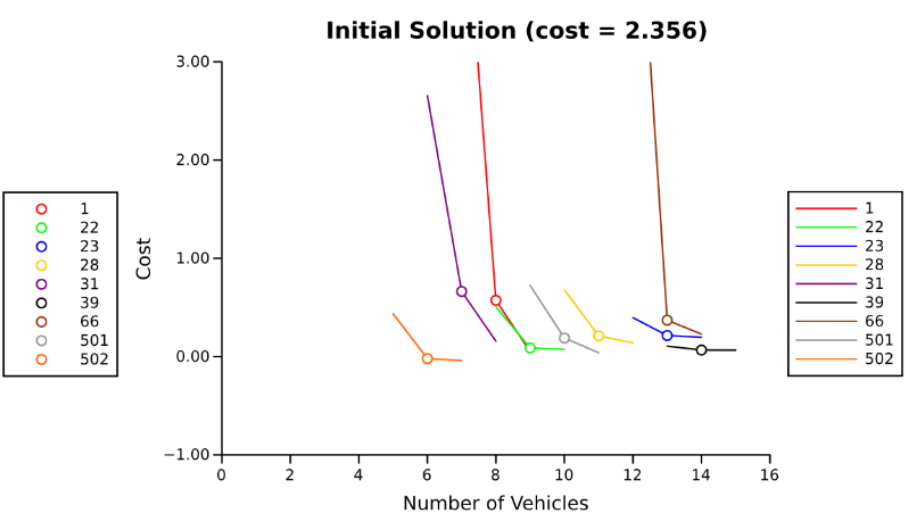

(b)

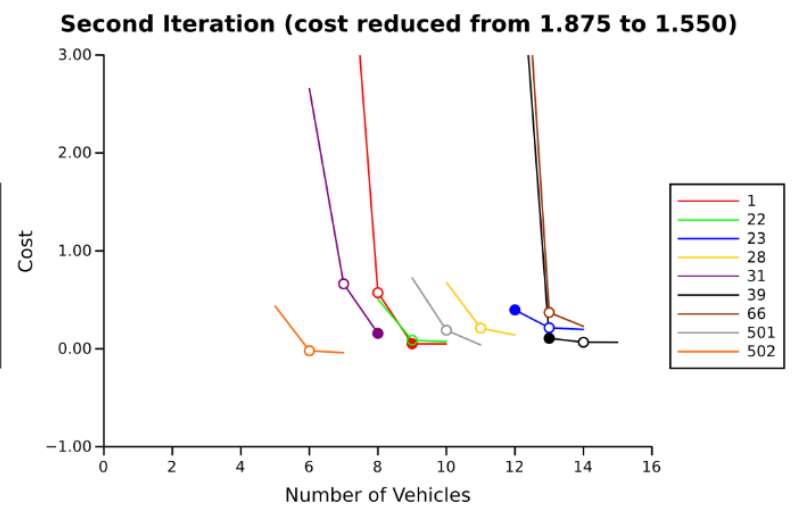

(d)

Figure 4: Fleet allocation optimization with nine MBTA morning peak bus services

The optimal allocation of vehicles shifts vehicles from routes with a small marginal increase in user cost to routes with relatively larger marginal decreases in user cost, with a tendency to equalize the derivatives of performance functions across routes. The resulting total decrease in (excess) user cost is 34\%. With five routes keeping their initial fleet size and four routes each losing or gaining one vehicle, the initial allocation is not far from optimal.

\section{Conclusion}

The overall budget-constrained service performance of a bus network can improve with the optimization of resource allocation. The strategy is to systematically move vehicles from routes in which they provide less benefit to routes in which they provide more benefit. It is not expected that the changes suggested by such an optimization routine will be dramatic; instead, they will most likely be small adjustments to an already reasonable allocation of resources. Three algorithms work together towards the optimal solution: an optimizer, a simulator, and a vehicle profiler. The objective function may contain a mixture of quantifiable aspects of service quality, such as excess waiting time and excess load. The optimizer acts on the objective function to decide where to remove and add resources. Since there are no real-life observations of performance under the hypothetical resource levels being investigated, estimates of these performance measures must be obtained; a simulation model based on the framework by Sánchez-Martínez (2012) is well-suited for the task. One 
of the challenges faced is the need to translate a single measure of fleet size into a realistic time-varying vehicle profile. Three simple strategies that modify an existing vehicle profile were presented.

In the application presented in Section 5, the allocation of vehicles to nine MBTA bus routes was optimized in order to minimize a combination of excess waiting time and excess load during morning peak operations. For simplicity, operations were simulated as a stationary process. Probability distributions of running times between timing points were obtained from one month of AVL data. Demand was modelled as a Poisson process with mean arrival rates calculated from AFC data, an origin-destination inference algorithm, and scaling. Small adjustments to fleet allocation resulted in a total excess user cost reduction of $34 \%$, keeping the total fleet size constant. Assuming a target headway dispatching policy at terminals, adding vehicles to a route increases the probability that successive vehicles are dispatched at, or close to, the target headway, but variable running times can lead to irregular headways downstream. Different control policies such as even headway dispatching and holding control at stops, as well as infrastructure upgrades such as dedicated lanes and conditional signal priority, could improve performance further.

Bus operators could employ an optimization framework like the one presented here to detect opportunities to improve service or save wasted resources, using automatically collected data. Although the optimization process is based on a pre-set objective function, detailed, disaggregate performance estimates can be part of the output in order to assist analysts in following up on automatically-generated suggestions.

Future work will focus on relaxing some of the simplifying assumptions made in the interest of tractability. Ridership models could be incorporated into the optimization model. Improvements in reliability and comfort can attract new ridership (and conversely, degraded reliability and comfort can drive away riders to other routes or modes), especially when a corridor is shared among multiple routes. Moreover, improved headway regularity can lower running times. Ridership shifts between routes serving a common corridor could be captured by considering multiple routes simultaneously in optimization and simulation, and modelling passenger route choice. A scheduling algorithm could also be incorporated into the framework in order to capture vehicle and crew constraints and to optimize allocation of resources throughout the day rather than for specific time periods. The framework developed in this research could be applied to optimize service frequencies in addition to fleet sizes by simulating the effect of changing the target dispatching headway and the number of vehicles operating. Alternatively, an even headway dispatching policy could be adopted to manipulate fleet size directly and let the headway emerge naturally from simulated operations.

\section{Acknowledgements}

This research was made possible by the National Science Foundation Graduate Research Fellowship Program under Grant No. 1122374, Transport for London, the Massachusetts Institute of Technology MISTI-PUC program, and the VREF BRT Centre of Excellence. 


\section{References}

Ceder, A., 1984. Bus frequency determination using passenger count data. Transportation Research Part A 18(5), 439-453.

Constantin, I., Florian, M., 1995. Optimizing frequencies in a transit network: a nonlinear bilevel programming approach. International Transactions in Operational Research 2(2), 149164.

Desaulniers, G., Hickman, M. D., 2007. Public transit. Handbooks in operations research and management science 14, 69-127.

Furth, P., Wilson, N. H. M., 1981. Setting frequencies on bus routes: theory and practice. Transportation Research Record 818, 1-7.

Gordon, J. B., Koutsopoulos, H. N., Wilson, N. H. M., Attanucci, J. P., 2013. Automated inference of linked transit journeys in London using fare-transaction and vehicle location data. Transportation Research Record 2343, 17-24.

Han, A., Wilson, N. H. M., 1982. The allocation of buses in heavily utilized networks with overlapping routes. Transportation Research Part B 16(3), 221-232.

Ibarra-Rojas, O. J., Delgado, F., Giesen, R., Muñoz, J. C., 2015. Planning, operation, and control of bus transport systems: a literature review. Transportation Research Part B 77, 3875.

Li, Y., Wangtu, X., Shiwei, H., 2013. Expected value model for optimizing the multiple bus headways. Applied Mathematics and Computation 219(11), 5849-5861.

Martínez, H., Mauttone, A., Urquhart, M., 2014. Frequency optimization in public transporation systems: formulation and metaheuristic approach. European Journal of Operational Research 236(1), 27-36.

Sánchez-Martínez, G. E., 2012. Running time variability and resource allocation: a datadriven analysis of high-frequency bus operations. Master Thesis. Massachusetts Institute of Technology.

Verbas, I., Mahmassani, H., 2013. Optimal allocation of service frequencies over transit network routes and time periods: formulation, solution and implementation using bus route patterns. Transportation Research Record 2334, 50-59.

Yu, B., Yang, Z., Yao, J., 2010. Genetic algorithm for bus frequency optimization. Journal of Transportation Engineering 136, 576-583. 